\title{
Uso de teléfonos inteligentes por alumnos de pregrado de Odontología durante la pandemia de COVID-19
}

\author{
Smartphone use by dental students during the COVID-19 pandemic
}
Jhon Paul lakov Mezarina Mendoza ${ }^{\text {labc }}$
Antuanett Olennka Chavez Valverde ${ }^{19}$
(iD) $h$ ttps://orcid.org/0000-0002-3496-2502
Joely Dominique Garrido Ayala ${ }^{19}$
Luis CarlosVélez Taboada ${ }^{\text {1ade }}$
(iD) https://orcid.org/0000-0001-5498-8673
María Fernanda Ortiz Vidal ${ }^{\text {if }}$
iD https://orcid.org/0000-0001-9737-1067
(iD) https://orcid.org/0000-0002-7418-8785
iD https://orcid.org/0000-0003-4413-330X

Correspondencia: jmezarinam@unmsm.edu.pe

\section{Resumen}

Las aplicaciones móviles emergentes pueden convertirse en instrumentos muy útiles para complementar las clases virtuales; sin embargo, aún no se aprovechan todas sus ventajas, porque no hay un diseño de estrategias establecido sobre cómo articular estas tecnologías en la educación. Objetivo: Determinar el uso de los teléfonos móviles por parte de los estudiantes de Odontología en tiempos de pandemia. Métodos: El diseño de la investigación fue descriptivo y transversal; se conformó por estudiantes ingresantes desde el año 2015 al 2020 de la Facultad de Odontología de la Universidad Nacional Mayor de San Marcos. Con un intervalo de confianza de $95 \%$, se seleccionaron 205 estudiantes que contaban con un teléfono inteligente y conexión a internet para responder la encuesta. Resultados: El $83.9 \%$ de los estudiantes instalaron aplicaciones móviles. Las actividades más frecuentes fueron: gestores de música ( $82.4 \%$ ), entretenimiento (66.3 \%), juegos educativos (40.5\%) y videoconferencias (37.1\%). Uno (26.3\%) o ningún docente $(25.4 \%)$ implementaron como herramienta metodológica las aplicaciones móviles. Sin embargo, se evidencia una buena percepción de la utilización de aplicaciones móviles, siendo que el $73.7 \%$ de los estudiantes está de acuerdo con su incorporación. Conclusión: La frecuencia de uso y la alta aceptación de uso promueven la implementación de aplicaciones de forma complementaria a la educación universitaria. Las instituciones deben promover su uso capacitando a los docentes y estudiantes sobre la elección y promoción de aplicaciones. inteligentes

Palabras clave: m-learning, educación médica, odontología, aplicaciones móviles, teléfonos

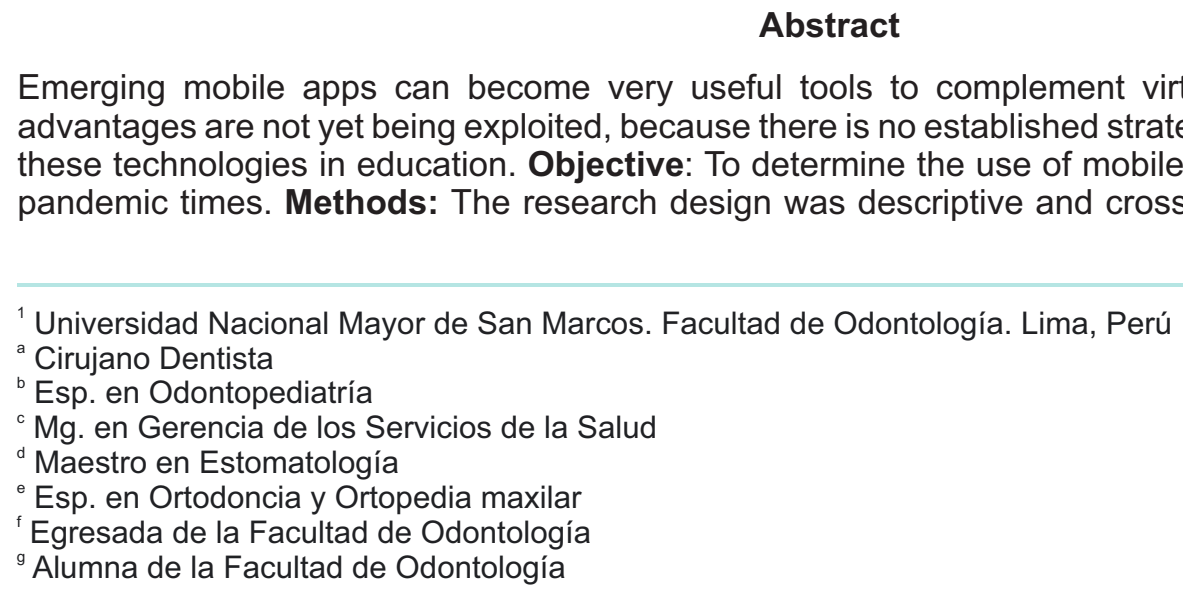

Abstract

\footnotetext{
${ }^{1}$ Universidad Nacional Mayor de San Marcos. Facultad de Odontología. Lima, Perú

${ }^{a}$ Cirujano Dentista

${ }^{b}$ Esp. en Odontopediatría

${ }^{\mathrm{c}} \mathrm{Mg}$. en Gerencia de los Servicios de la Salud

${ }^{\mathrm{d}}$ Maestro en Estomatología

${ }^{\text {e }}$ Esp. en Ortodoncia y Ortopedia maxilar

${ }^{f}$ Egresada de la Facultad de Odontología

${ }^{g}$ Alumna de la Facultad de Odontología
}

Emerging mobile apps can become very useful tools to complement virtual classes. However, all its advantages are not yet being exploited, because there is no established strategy design on how to articulate these technologies in education. Objective: To determine the use of mobile phones by dental students in pandemic times. Methods: The research design was descriptive and cross-sectional. It was made up of 
incoming students from 2015 to 2020 from the Faculty of Dentistry of the Universidad Nacional Mayor de San Marcos. With a $95 \%$ confidence interval, 205 students who had a smartphone and an internet connection were selected to answer the survey. Results: $83.9 \%$ of the students installed mobile applications. The most frequent activities were music managers (82.4\%), entertainment $(66.3 \%)$, educational games $(40.5 \%)$ and videoconferences $(37.1 \%)$. One $(26.3 \%)$ or no teachers $(25.4 \%)$ used mobile applications as a methodology tool. However, a good perception of the use of mobile apps is evidenced, with $73.7 \%$ of students agreeing with their incorporation. Conclusion: The frequency of use and the high acceptance of use promote the implementation of applications in a complementary way to university education. Institutions should promote its use by training teachers and students on the choice and promotion of apps.

Keywords: m-learning, medical education, dentistry, apps, smartphones

\section{Introducción}

En diciembre del 2019 se reportó el primer paciente con COVID-19 en Wuhan (China), descubriéndose una nueva variante, el SARS CoV-2. ${ }^{1}$ Su elevada morbilidad y rápida expansión promovió que la Organización Mundial de la Salud (OMS) declare a la COVID-19 como emergencia de salud pública de importancia internacional ${ }^{2}$ y con ello la necesidad de implementar protocolos y restricciones a nivel estatal.

En el Perú, mediante Resolución Viceministerial 081-2020-MINEDU se decide la postergación del inicio de actividades lectivas en las universidades públicas y privadas. ${ }^{3}$ Ante ello, con Resolución Rectoral N¹269-R-20, la Universidad Nacional Mayor de San Marcos (UNMSM) aprueba el Cronograma de Actividades Académicas No Presenciales como estrategia pedagógica adaptativa a la emergencia nacional. ${ }^{4-6}$

Las tecnologías de información y comunicación (TIC) utilizan servicios que intercambian información; entre ellas se incluyen a las páginas web, redes sociales, aplicaciones en teléfonos inteligentes, entre otros. Estas herramientas, incluidas las pedagógicas, brindan apoyo a procesos metodológicos ${ }^{7,8}$ que, después de un tiempo, se orientarán hacia las poblaciones con limitaciones de acceso a la educación en diferentes niveles. ${ }^{9}$

El acceso mundial a internet en el año 2020 fue en aumento. Actualmente, se considera que al menos un miembro de la familia cuenta con un smartphone ${ }^{10}$ además, se ha evidenciado un aumento tanto en acceso como en innovaciones tecnológicas. La digitalización y la robótica son consideradas la cuarta revolución industrial, permitiendo que sus características sean implementadas en el aprendizaje. ${ }^{11}$

En el contexto actual de la pandemia, las aplicaciones móviles emergentes pueden convertirse en instrumentos muy útiles para fomentar los cuidados en salud; en el campo educativo, su uso es un recurso para complementar las clases virtuales. ${ }^{12}$ Por ese motivo, el uso de internet como la telefonía móvil son pilares básicos en la revolución social y académica de los jóvenes. ${ }^{13}$ Actualmente, el docente no promueve el uso del teléfono inteligente o smartphone; en tal sentido, no hay un diseño de estrategias de cómo articular estas tecnologías en la educación que logre que el smartphone sea una herramienta de apoyo a la enseñanza y así aprovechar todas sus ventajas. ${ }^{12}$

La presente investigación tiene como finalidad determinar cuál es el uso de los teléfonos móviles por parte de los estudiantes en tiempos de pandemia. 


\section{Método y material}

El presente trabajo de investigación tiene un diseño descriptivo y transversal. La muestra está conformada por estudiantes de la Facultad de Odontología de la Universidad Nacional Mayor de San Marcos que se encuentren matriculados en el semestre académico 2020 - II, que cuenten con matrícula vigente y sean alumnos regulares. Son considerados para la investigación los ingresantes desde el año 2015 al 2020. Los participantes debieron contar con un teléfono inteligente, o smartphone, con conexión a internet para poder acceder al cuestionario electrónico.

El tamaño de la muestra fue determinado a partir de una población de 365 estudiantes, con un $95 \%$ de confianza, precisión de $5 \%$ y proporción de las variables de interés de $50 \%$; que resultaron en un tamaño muestral mínimo de 179. Teniendo en cuenta el ajuste de pérdidas de $10 \%$ se seleccionó aleatoriamente a 205 estudiantes.

La recolección de datos se llevó a cabo con una encuesta sobre el uso de dispositivos móviles tomada del artículo "Percepción del uso educativo del teléfono inteligente en estudiantes de la Universidad de la Sierra Sur", de la autora Aidee Cruz Barragán, disponible en Temas de Ciencia y Tecnología. ${ }^{14} \mathrm{El}$ instrumento cuenta con 11 preguntas con respuestas dicotómicas (sí y no) que nos permitieron la identificación de variables como sexo, año que se encuentra cursando, si cuenta con un smartphone, disponibilidad de internet en su dispositivo, el tipo de conexión que utiliza, la actividad principal que realiza en su smartphone, instalación de aplicaciones (app), si utiliza aplicaciones y estas le ayudan en su formación educativa, número de profesores que hacen uso de estas aplicaciones y, por último, las aplicaciones más usadas por los alumnos. La encuesta se desarrolló en la plataforma $\mathrm{G}$ suite de Google y fue enviada a los correos institucionales, además de tener como soporte la comunicación directa por la aplicación WhatsApp; todos los participantes involucrados en la recolección de datos accedieron voluntariamente a participar en el estudio.

Para el análisis estadístico descriptivo se emplearon tablas de frecuencia con el programa estadístico SPSS 24.0.

\section{Resultados}

205 estudiantes participaron en el estudio de investigación. El sexo femenino se presentó con mayor frecuencia (61.5\%), siendo casi 3 veces mayor a la frecuencia del sexo masculino (38.5\%). La edad de los participantes fue distribuida en 4 grupos; el de mayor frecuencia fue el grupo entre los 21 y 24 años (61\%), seguido por el grupo entre los 17 y 20 años (23.9\%), luego el grupo entre los 25 y 28 años $(14.1 \%)$ y, por último, solo el $1 \%$ tiene más de 28 años. Se observó mayor participación de los alumnos de $4 .^{\circ}$ año $(27.3 \%)$, seguidos por alumnos de $2 .^{\circ}$ año $(20 \%), 6 .^{\circ}$ año $(19 \%), 3$. $^{\text {er }}$ año $(17.6 \%)$ y, por último, de $5 .^{\circ}$ año (16.1\%). El $97.6 \%$ de la población cuenta con acceso a internet mediante el uso de wifi (56.2 \%) y plan de datos móviles contratados (41.9\%). Solo un $2 \%$ de la población no cuenta con un teléfono inteligente y 172 alumnos (83.9\%) instalan aplicaciones en el teléfono inteligente (Tabla 1).

Se observó que el uso de aplicaciones educativas por alumnos del $4 .^{\circ}$ año $\left(30.4 \%\right.$ ) y $6 .{ }^{\circ}$ año (46.2\%) es más frecuente. De acuerdo al tipo de aplicaciones utilizadas, el uso de aplicaciones musicales (82.4\%) es de mayor frecuencia, seguidas por las de entretenimiento (66.3\%), manejo de documentos (64.4\%), juegos educativos (40.5\%), videoconferencias (37.1\%) y de odontología $(34.1 \%)$, respectivamente (Tabla 2$)$. 
Se evidencia que, por lo menos, el $25.4 \%$ de los alumnos tienen docentes que no indican el uso de aplicaciones educativas como herramienta para la formación académica, mientras que el 21 $\%$ manifiesta que la mayoría de sus profesores piden el uso de aplicaciones educativas. El $73.7 \%$ de la población de estudio considera que las aplicaciones educativas ayudan mucho si se usan adecuadamente (Tabla 3 ).

\section{Tabla 1}

Características demográficas y uso de internet

\begin{tabular}{|c|c|c|c|}
\hline Variables & Años & $\mathrm{N} .^{\circ}$ & $\%$ \\
\hline \multirow{4}{*}{ Edad } & $17-20$ & 49 & 23.9 \\
\hline & $21-24$ & 125 & 61 \\
\hline & $25-28$ & 29 & 14.1 \\
\hline & más de 28 & 2 & 1 \\
\hline \multirow{2}{*}{ Género } & Femenino & 126 & 61.5 \\
\hline & Masculino & 79 & 38.5 \\
\hline \multirow{5}{*}{ Año de estudio } & $2 .^{\circ}$ & 41 & 20 \\
\hline & $3 .^{\circ}$ & 36 & 17.6 \\
\hline & $4 .^{\circ}$ & 56 & 27.3 \\
\hline & $5 .^{\circ}$ & 33 & 16.1 \\
\hline & $6 .^{\circ}$ & 39 & 19 \\
\hline \multirow{2}{*}{$\begin{array}{l}\text { Tenencia de } \\
\text { teléfono inteligente }\end{array}$} & Sí & 201 & 98 \\
\hline & No & 4 & 2 \\
\hline \multirow{2}{*}{ Acceso a internet } & Sí & 200 & 97.6 \\
\hline & No & 5 & 2.4 \\
\hline \multirow{3}{*}{$\begin{array}{c}\text { Tipo de acceso } \\
\text { a internet }\end{array}$} & $\begin{array}{c}\text { Datos móviles } \\
\text { contratados }\end{array}$ & 86 & 41.9 \\
\hline & Wifi & 115 & 56.2 \\
\hline & Sin acceso & 4 & 1.9 \\
\hline \multirow{2}{*}{$\begin{array}{l}\text { Instalación de } \\
\text { aplicaciones }\end{array}$} & Sí & 172 & 83.9 \\
\hline & No & 33 & 16.1 \\
\hline
\end{tabular}


Tabla 2

Uso de aplicaciones educativas

\begin{tabular}{|c|c|c|c|c|c|}
\hline \multirow{2}{*}{ Variable } & \multirow{2}{*}{ Año } & \multicolumn{2}{|c|}{ Sí } & \multicolumn{2}{|c|}{ No } \\
\hline & & $\mathrm{N}^{\circ}$ & $\%$ & $\mathrm{~N} .^{\circ}$ & $\%$ \\
\hline \multirow{5}{*}{ Año } & $2 .^{\circ}$ & 14 & 34.1 & 27 & 65.9 \\
\hline & $3 .{ }^{\circ}$ & 13 & 36.1 & 23 & 63.9 \\
\hline & $4 .^{\circ}$ & 17 & 30.4 & 39 & 69.6 \\
\hline & $5 .{ }^{\circ}$ & 11 & 33.3 & 22 & 66.7 \\
\hline & $6 .^{\circ}$ & 18 & 46.2 & 21 & 53.8 \\
\hline \multirow{6}{*}{ Tipo de aplicaciones } & Música & 169 & 82.4 & 36 & 17.6 \\
\hline & Entretenimiento & 136 & 66.3 & 69 & 33.7 \\
\hline & Juegos educativos & 83 & 40.5 & 122 & 59.5 \\
\hline & Videoconferencias & 76 & 37.1 & 129 & 62.9 \\
\hline & Documentos & 132 & 64.4 & 73 & 35.6 \\
\hline & Odontología & 70 & 34.1 & 135 & 65.9 \\
\hline $\begin{array}{c}\text { Sin acceso a } \\
\text { internet }\end{array}$ & - & 73 & 35.6 & 132 & 64.4 \\
\hline
\end{tabular}

Tabla 3

Recurso académico

\begin{tabular}{|c|c|c|c|}
\hline \multirow{2}{*}{ Variable } & Docente & $\mathrm{N}^{\circ}$ & $\%$ \\
\hline \multirow{4}{*}{ Pedido por } & Mayoría & 43 & 21 \\
\cline { 2 - 4 } & 2 o 3 & 56 & 27.3 \\
\cline { 2 - 4 } & 1 & 54 & 26.3 \\
\hline \multirow{3}{*}{$\begin{array}{c}\text { Herramienta de } \\
\text { apoyo }\end{array}$} & Ninguno & 52 & 25.4 \\
\cline { 2 - 4 } & No ayuda & 3 & 1.5 \\
\cline { 2 - 4 } & Un poco & 51 & 24.9 \\
\hline \multirow{4}{*}{$\begin{array}{c}\text { Mucho, si se usa } \\
\text { adecuadamente }\end{array}$} & 151 & 73.7 \\
\hline
\end{tabular}

\section{Discusión}

Se realizó la encuesta a 205 estudiantes de la Facultad de Odontología matriculados en un semestre académico, donde 126 encuestados pertenecen al género femenino $(61.5 \%)$ y 79 al género masculino (38.5\%). El promedio de edad de los encuestados fue de 20.6 años, siendo el rango de 21 a 24 años (61\%) el de mayor frecuencia.

La aceptación de las aplicaciones móviles se relaciona con su facilidad de uso y la costumbre digital de los estudiantes, permitiendo así compartir directamente materiales didácticos. Este 
estudio evidencia que solo el $35.6 \%$ de los 205 estudiantes con teléfonos inteligentes utilizan aplicaciones educativas, siendo un indicador de la desactualización en cuanto a los beneficios de los Smartphone. Estos resultados son ligeramente menores que los obtenidos por Mejía en su investigación realizada en la serranía peruana (39.9\%) a estudiantes de Medicina. ${ }^{15}$

Nuestra encuesta evidenció que el $98 \%$ de los estudiantes cuenta con un teléfono inteligente. Suner obtuvo resultados similares a los nuestros; el $97.5 \%$ de sus encuestados cuentan con un teléfono inteligente; el $23.5 \%$, con una computadora y el $85.2 \%$ cuentan con ambos dispositivos. ${ }^{16}$

Dafonte et al. aplicaron un cuestionario a 179 estudiantes universitarios, evidenciando que el dispositivo de preferencia es la laptop, seguido por el teléfono inteligente, pero este último cuenta con mayor acceso estudiantil. El uso de los móviles es aprobado por el $89.77 \%$ como bastante útil, siendo las actividades más frecuentes los servicios de alerta de entrega, campus virtual para gestionar información del docente, aplicaciones de mensajería para contactar con los docentes, aplicaciones de educación colaborativa como foros o cuestionarios didácticos, reproducir clases grabadas, entre los más frecuentes. En nuestro estudio, el porcentaje de aceptación también es alto $(73.7 \%)$, pero los participantes consideran que debe usarse adecuadamente para complementar la formación académica; las aplicaciones educativas con fines odontológicos de mayor uso son las que organizan documentos y acceso a videoconferencias. ${ }^{17}$

Una de las fortalezas e interés por la incorporación de aplicaciones móviles es que se ha popularizado su uso siendo una herramienta habitual e innovadora en la mayoría de los estudiantes actuales. Un ensayo aleatorio evaluó la diferencia entre una estrategia tradicional y una digital; se obtuvo que los conocimientos cognitivos fueron estadísticamente similares. Sin embargo, la motivación y satisfacción general fue mayor en el grupo intervenido. Además, el grupo control manifestó mayores índices de ira, hostilidad y de confusión en comparación de la media del grupo intervenido. ${ }^{18}$ Extrapolamos estos resultados como fundamentos de la percepción e interés de los estudiantes encuestados para la incorporación de aplicaciones en la estrategia educativa universitaria. Uno de los temas a analizar es la incorporación obligatoria de aplicaciones, ya que nuestro estudio evidenció que un $2.4 \%$ no presenta conexión a internet.

Un cuarto de los estudiantes (25.4\%) manifestaron que ningún profesor incorporó aplicaciones móviles como estrategia de aprendizaje, aperturando dos interrogantes. La primera, sobre los conocimientos del docente de proporcionar o crear aplicaciones educativas y, por otro lado, la capacidad de los estudiantes para discernir entre el material educativo virtual basado en evidencia, ya que un 83.9 \% utiliza aplicaciones con objetivos académicos. Caravelli evidenció que la incorporación de la enseñanza virtual es más sólida y aceptada cuando la universidad proporciona condiciones facilitadoras. ${ }^{19}$ A la vez, en un estudio realizado en el 2019 a estudiantes de Odontología, se resaltó que la principal problemática es la elección del material, debido a la variedad de propuestas en la red..$^{20}$

\section{Conclusiones}

Se concluye que el $98 \%$ de los estudiantes de la Facultad de Odontología de la UNMSM cuentan con un smartphone; y que el $97.6 \%$ de los estudiantes cuentan con acceso a internet. Las aplicaciones móviles fueron de gran aceptación por los estudiantes, realizando diversas actividades como de entretenimiento y educativas. 
La frecuencia de uso y su alta aceptación promueven la implementación de aplicaciones de forma complementaria a la educación universitaria.

\section{Referencias}

1. Organización Panamericana de la Salud / Organización Mundial de la Salud. Actualización Epidemiológica: Nuevo coronavirus (COVID - 19). Washington, DC OPS/OMS. 2020.

2. OPS/OMS [Internet]. La OMS caracteriza a COVID-19 como una pandemia. 2020 [citado el 3 de abril del 2020]. Disponible en: https://www.paho.org/

3. Plataforma Digital Única del Estado Peruano. Resolución Viceministerial N. ${ }^{\circ}$ 081-2020MINEDU [Internet]. Lima: MINEDU; 2020 [citado 27 agosto 2020]. Disponible en: https://cdn.www.gob.pe/uploads/document/file/565533/RVM_N_081-2020-MINEDU.PDF

4. Universidad Nacional Mayor de San Marcos. Resolución Rectoral N. ${ }^{\circ}$ 01269-R-20 [Internet]. Lima: UNMSM; 2020 [citado 27 agosto 2020]. Disponible en: http://previous.unmsm.edu.pe/transparencia/archivos/01269-R-20-anexos.pdf

5. Universidad Nacional Mayor de San Marcos. Resolución Rectoral N. ${ }^{\circ}$ 01317-R-20 [Internet]. Lima: UNMSM; 2020 [citado 27 agosto 2020]. Disponible en: http://previous.unmsm.edu.pe/transparencia/archivos/01317-R-20.pdf

6. Universidad Nacional Mayor de San Marcos. Resolución Rectoral N. ${ }^{\circ}$ 016786-2020R/UNMSM [Internet]. Lima: UNMSM; 2020 [citado 27 agosto 2020]. Disponible en: http://previous.unmsm.edu.pe/transparencia/archivos/016786.pdf

7. McGowan JJ. Commentary: information technologies and the transformation of nursing education. Nurs Outlook. 2008 Sep-Oct;56(5):231. doi: 10.1016/j.outlook.2008.04.006. PMID: 18922274.

8. Gasca Hurtado G., Machuca Villegas, L. El desarrollo de software como habilitador digital para la 4ta revolución industrial. RISTI [online]. 2020, n.39 [citado 2021-02-16], págs. 11-16 D i s p o n i b l e http://www.scielo.mec.pt/scielo.php?script=sci_arttext\&pid=S164698952020000400001\&l ang=es doi: http://dx.doi.org/10.17013/risti.39.0.

9. Gassert CA. Technology and informatics competencies. Nurs Clin North Am. 2008 Dec;43(4):507-21, v. doi: 10.1016/j.cnur.2008.06.005. PMID: 18940408.

10. Graf César. Tecnologías de información y comunicación (TICs). Primer paso para la implementación de TeleSalud y Telemedicina. Rev. parag. reumatol. [Internet]. 2020 June [ cited $2021 \mathrm{~F} \mathrm{e} \mathrm{b} \mathrm{16]} \mathrm{;} \mathrm{6(} \mathrm{1):} \mathrm{1-4.} \mathrm{Disponible} \mathrm{en:}$ http://scielo.iics.una.py/scielo.php?script=sci_arttext\&pid=s241343412020000100001\&lng=en. https://doi.org/10.18004/rpr/2020.06.01.1-4.

11. Bensusán Areous G, Eichhorst W, Rodríguez J. Las transformaciones tecnológicas y sus desafíos para el empleo, las relaciones laborales y la identificación de la demanda de cualificaciones. Santiago: CEPAL; 2017.

12. Galindo Neto Nelson Miguel, Sá Guilherme Guarino de Moura, Barbosa Luciana Uchôa, Pereira Juliana de Castro Nunes, Henriques Amanda Haissa Barros, Barros Lívia Moreira. COVID-19 AND DIGITAL TECHNOLOGY: MOBILE APPLICATIONS AVAILABLE FOR DOWNLOAD IN SMARTPHONES. Texto contexto - enferm. [Internet]. 2020 [cited 2021 $\mathrm{F} \mathrm{e} \mathrm{b} 16$ ] ; 29 : e 20200150 . A v a ila ble from: http://www.scielo.br/scielo.php?script=sci_arttext\&pid=S010407072020000100203\&lng=en. Epub July 06, 2020. https://doi.org/10.1590/1980-265xtce-2020-0150. 
13. Ruiz-Palmero Julio, Sánchez-Rodríguez José, Trujillo-Torres Juan Manuel. Utilización de Internet y dependencia a teléfonos móviles en adolescentes. Rev.latinoam.cienc.soc.niñez juv [Internet]. 2016 July [cited 2021 Feb 16] ; 14( 2 ): 1357-1369. Available from: http://www.scielo.org.co/scielo.php?script=sci_arttext\&pid=S1692 715X2016000200033\&lng=en. http://dx.doi.org/10.11600/1692715x.14232080715.

14. CruzA, Barragán A. Percepción del uso educativo del teléfono inteligente en estudiantes de la Universidad de la Sierra Sur. TEMAS DE CIENCIAY TECNOLOGÍA.2017; 21(61), 29-40.

15. Mejia Christian R, Flores Salder D, Verastegui-Díaz Araseli, García-Moreno Katerine M, Vargas Mariela, Cárdenas Matlin M et al. Uso del Smartphone y de Facebook asociado a la autopercepción del rendimiento académico en estudiantes de Medicina peruanos. Rev. cuba. inf. cienc. salud [Internet]. 2017 Mar [citado 2021 Feb 09]; 28(1):77-88. Disponible en: http://scielo.sld.cu/scielo.php? script=sci_arttext\&pid=S230721132017000100006\&lng=es.

16. Suner A, Yilmaz Y, Pişkin B. Mobile learning in dentistry: usage habits, attitudes and perceptions of undergraduate students. PeerJ. 2019 Jul 29;7: e7391. Doi:10.7717/peerj.7391U

17. Dafonte Gómez A, Maina MF, García Crespo O. Smartphone use in university students: An opportunity for learning. Pixel-Bit, Rev Medios Educ 2021(60):211-227. Recuperado de: https: // www.scopus.com/record/display.uri? eid=2-s $2.0-$ $85098946703 \&$ or i g i n $=r$ es u It s list \& sort $=p \mid f-$ $\mathrm{f} \& \mathrm{src}=\mathrm{s} \& \mathrm{st} 1=\& \mathrm{st} 2=\& \mathrm{sid}=\mathrm{df} 8 \mathrm{f} 19 \mathrm{~cd} 6 \mathrm{ad} 55049 \mathrm{~d} 4 \mathrm{c} 56 \mathrm{fc} 0 \mathrm{~d} 54 \mathrm{ca} 8 \mathrm{~b} 4 \& \mathrm{sot}=\mathrm{b} \& \mathrm{sdt}=\mathrm{b} \& \mathrm{sl}=19 \& \mathrm{~s}=\mathrm{F}$ IRSTAUTH+\%28Dafonte\%29\&relpos=0\&citeCnt=1\&searchTerm=

18. Lozano Lozano M, Fernández Lao C, Cantarero-Villanueva I, Noguerol I, Álvarez-Salvago F, Cruz-Fernández M, et al. A blended learning system to improve motivation, mood state, and satisfaction in undergraduate students: Randomized controlled trial. J Med Internet Res 2020;22(5). Recuperado de: https://www.scopus.com/record/display.uri?eid=2-s2.085085537815 \& or i g i $n=r$ e s u l t s I i s t \& sort $=p \mid f-$ f\&src=s\&st1 =\&st2=\&sid=0d69942852642a70fbdccfc54fa09519\&sot=b\&sdt=b\&sl=57\&s=T I $T$ L E - A B S - K E Y + \% 28 M LEARNING+AND+test+AND+medical+education\%29\&relpos=1\&citeCnt=2\&searchTerm=

19. Carabelli, Patricia. "Response to the COVID-19 Outbreak: Virtual Teaching Time." InterCambios. Dilemas y transiciones de la Educación Superior 7 (2020): 189-198.

20. Khatoon B, Hill K, Walmsley AD. Mobile learning in dentistry: challenges and opportunities. Brit Dent J 2019;227(4):298-304.

Conflicto de intereses: Esta investigación no presenta conflicto de intereses entre los investigadores.

Fuente de financiamiento: La presente investigación fue financiada por los investigadores. 